\title{
UNIVALENCE OF HOLOMORPHIC MAPPINGS
}

\author{
Robert Molzon and Karen Pinney Mortensen
}

\begin{abstract}
Univalence of holomorphic mappings is studied via two differential operators naturally associated with the mapping. The first operator is invariant under composition on the left with a projective linear mapping and the second operator is invariant under composition with holomorphic Euclidean transformations. The methods used are analogous to methods used by Osgood and Stowe in the case of conformal mappings.
\end{abstract}

\section{Introduction.}

In this paper, we consider the role played by certain differential operators in the question of univalence of holomorphic mappings. In an earlier paper [4] we presented several equivalent ways of associating two differential operators with a holomorphic mapping, and we showed how these operators were analogous to the classical Schwarzian derivative. The classical result of Nehari [6] gives a sufficient condition for a holomorphic function defined on the disk to be univalent; the condition is a bound on the Schwarzian derivative. Recently, M. Chuaqui [3] presented a uniform approach to univalence criteria for holomorphic functions on simply connected domains in the complex plane. Chuaqui's approach is based on the geometric ideas developed by Osgood and Stowe [7] in their generalization of the Schwarzian operator for conformal mappings. A very thorough survey of the classical univalence criteria for holomorphic functions is given in a paper by Avkhadiev and Aksent'ev [1]. This survey briefly mentions the question of univalence in higher dimensions.

There are several published results on univalence for holomorphic mappings. A sufficient condition for univalence of a mapping $f: \mathbf{C}^{n} \rightarrow \mathbf{C}^{n}$ in terms of the variation of the partial derivatives of the component functions was given by Takahashi [9]. A more subtle criterion was given by Pfaltzgraff [8] in which an extension of the classical one variable method of subordination chains was used to obtain sufficient conditions for univalence. Pfaltzgraff used a mapping of the form

$$
(z, \omega) \longmapsto(z, \omega+g(z))
$$


to show that his sufficient condition for univalence is not necessary. It also shows that the sufficient condition for univalence given by Takahashi is not necessary. Indeed, this example indicates that it may be very difficult to find simple necessary conditions for univalence based on bounds on derivatives of the mapping.

A criterion for univalence of conformal mappings between Riemannian manifolds was obtained recently by Osgood and Stowe [7] and also by Carne [2]. In both of these papers, the criterion for univalence is stated in terms of bounds on operators analogous to the Schwarzian derivative. Osgood and Stowe derive their univalence result by considering a function which satisfies a differential inequality along geodesics in the domain of the mapping. Their Schwarzian operator appears as a coefficient in the differential inequality.

In this paper we follow the approach used by Osgood and Stowe, and obtain a differential inequality along geodesics. In our case the differential inequality is somewhat more complicated, and involves two operators associated with a holomorphic mapping. The two operators together play a role analogous to the Schwarzian; our univalence criteria are stated in terms of bounds for both operators.

Although we state one particular univalence criterion in Theorem 3.1, it will be clear from our proof that other results along this line are possible using the same method. In particular Equation (3.3) provides a general method of deriving univalence criteria from two differential operators associated with a holomorphic mapping.

\section{Background.}

In this section we present two differential operators associated with a holomorphic mapping. These operators were studied in detail in our earlier paper [4], and play a role analogous to the Schwarzian derivative. They will be used to study injectivity criteria.

We shall be primarily interested in holomorphic mappings of domains $\Omega \subseteq$ $\mathbf{C}^{n}$ to either $\mathbf{C}^{n}$ or $\mathbf{C P}^{n}$. The first operator we define, $\mathcal{S}$, is a projectively invariant operator, and although defined with respect to specific choices of connection on domain and range, depends only on the projective class of the connection. Let $f: \Omega \rightarrow \mathbf{C P}^{n}$ be a locally biholomorphic mapping. Let $D$ denote the standard Euclidean connection on $\Omega$ and let $\nabla$ denote any connection complex projectively equivalent to the standard invariant connection on $\mathbf{C P}^{n}$. In particular one can take $\nabla$ to be the Levi-Civita connection associated with the Fubini-Study metric (which we refer to as the F-S connection). All connections will be of type $(1,0)$ and we therefore assume our vector fields to be of type $(1,0)$ unless otherwise noted. Define 
the difference tensor $\mathcal{D}$ by

$$
\mathcal{D}(X, Y)=f_{*}\left(D_{X} Y\right)-\nabla_{f_{*}} f_{*} Y
$$

for $X, Y \in \Gamma\left(\Omega, T^{1,0} \Omega\right)$. Since $f$ is assumed to be locally biholomorphic we can apply $f_{*}^{-1}$ to $\mathcal{D}(X, Y)$ and obtain a vector field on $\Omega$,

$$
\mathcal{D}_{\Omega}(X, Y)=f_{*}^{-1} \mathcal{D}(X, Y) .
$$

The operator $\mathcal{S}$ is defined as the trace free part of $\mathcal{D}_{\Omega}$. It is possible to express $\mathcal{S}$ as a measure of the failure of the push forward of the Euclidean connection to be projectively equivalent to the F-S connection on $\mathbf{P}^{n} \mathbf{C}$. Let $\nu_{F S}$ denote the volume form of the Fubini-Study metric, and $\nu_{E}$ the volume form of the Euclidean metric on $\Omega$. Define a $(1,0)$ form $\omega$ by

$$
\omega=\frac{1}{n+1} \partial \log \left\|f^{*} \nu_{F S}\right\|
$$

where the norm is defined with respect to the form $\nu_{E}$. Then $\mathcal{S}$ is given by

$$
\mathcal{S}(X, Y)=\mathcal{D}_{\Omega}(X, Y)-\omega(X) Y-\omega(Y) X
$$

which is the trace free part of $\mathcal{D}_{\Omega}$.

For $n \geq 2$ we define a third order differential operator $L$ by

$$
L=\frac{1}{n-1} \operatorname{tr}\left(D \mathcal{S}-\mathcal{S} \otimes_{t} \mathcal{S}\right)
$$

where $\otimes_{t}$ denotes the trace of the tensor product. The symmetry of $\Sigma$ implies that all traces of $\Sigma \otimes \Sigma$ are identically zero but one, and it is this trace we denote by $\otimes_{t}$. The operator $L$ is an element of $T^{1,0} \Omega \otimes T^{1,0} \Omega$ and is invariant under a projective change of connection on $\Omega$.

The operators $\mathcal{S}$ and $L$ appear in a natural way in terms of the canonical lift defined below of the mapping $f$. Let $\left[Z^{0}: Z^{1}: \ldots: Z^{n}\right]$ denote homogeneous coordinates on $\mathbf{C P}^{n}$ and let $U_{0} \subset \mathbf{C P}^{n}$ denote the domain with $Z_{0} \neq 0$. Define

$$
J=\operatorname{det}\left(\frac{\partial \varsigma^{\alpha}}{\partial z^{i}}\right)
$$

where $z=\left(z^{1}, \cdots, z^{n}\right)$ denote standard complex Euclidean coordinates on $\Omega$ and $\varsigma^{\alpha}=f^{\alpha}(z)$ denote standard nonhomogeneous coordinates on $U_{0}$. The canonical lift, $\tilde{f}$, of $f$ over $U_{0}$ is then given by

$$
\begin{gathered}
\tilde{f}: \Omega \rightarrow \mathbf{C}^{n+1}-\{0\} \\
\tilde{f}(z)=\left(\lambda(z), \lambda(z) f^{1}(z), \cdots, \lambda(z) f^{n}(z)\right)
\end{gathered}
$$


where

$$
\lambda^{n+1}(z) J(z) \equiv 1
$$

If $\pi: \mathbf{C}^{n+1}-\{0\} \rightarrow \mathbf{C P}^{n}$ is the canonical projection, then $\pi \circ \tilde{f}=f$. Let $Z=\left(Z^{0}, \cdots, Z^{n}\right)$ denote complex Euclidean coordinates on $\mathbf{C}^{n+1}-\{0\}$. Let $e_{i}=\tilde{f}_{*} \partial / \partial z^{i}$. The coefficients of $\mathcal{S}$ and $L$ can be obtained from the covariant derivatives of the $e_{i}$. If

$$
\begin{aligned}
& \mathcal{S}=\mathcal{S}_{i j}^{k} \partial / \partial z^{k} \otimes d z^{i} \otimes d z^{j} \quad \text { and } \\
& L=L_{i j} d z^{i} \otimes d z^{j}
\end{aligned}
$$

then

$$
D_{e_{i}} e_{j}=-L_{i j} \epsilon+\mathcal{S}_{i j}^{k} e_{k}
$$

Here $D$ denotes the Euclidean connection on $\mathbf{C}^{n+1}$ and

$$
\epsilon=Z^{0} \partial / \partial Z^{0}+\cdots+Z^{n} \partial / \partial Z^{n}
$$

is the Euler vector field on $\mathbf{C}^{n+1}-\{0\}$. Equation (2.1) will provide a convenient way to derive the differential inequality for univalence.

In order to derive the univalence criteria, we shall need an auxiliary function that maps $\mathbf{C P}^{n}$ to the nonnegative real numbers similar to the function used by Osgood and Stowe in obtaining their univalence criterion.

Let $P_{0}$ denote the point $[1: 0 \ldots: 0]$ in the homogeneous coordinates on $\mathbf{C P}^{n}$. Define

$$
W(Z)=\frac{\left|Z^{1}\right|^{2}+\ldots+\left|Z^{n}\right|^{2}}{\left|Z^{0}\right|^{2}+\ldots+\left|Z^{n}\right|^{2}} .
$$

Let $P_{0}$ denote any other point of $\mathbf{C P}^{n}$ and let $\varphi_{P}$ be an isometry of $\mathbf{C P}^{n}$ taking $P$ to $P_{0}$. Define $W_{P}=W \circ \varphi_{P}$. The function $W$ was used in [5] to characterize $\mathbf{C P}^{n}$ with the Fubini-Study metric up to biholomorphic isometry. The characterization was provided by the existence of a solution of a nonlinear partial differential equation on a hermitian manifold. On $\mathbf{C P}^{n}$ the solution to this equation is essentially given by $W$. In their extension of Nehari's univalence criteria to the conformal case, Osgood and Stowe made use of an analogous function and partial differential equation.

\section{The Univalence Criteria.}

We shall now develop a differential inequality along geodesics (lines) in $\Omega$ which will give a sufficient condition for injectivity of $f$. The basic idea of the derivation of the inequality follows Osgood and Stowe [7]. Our main result on univalence is as follows. 
Theorem 3.1. Let $\Omega \subset \mathbf{C}^{n}$ be a connected convex domain of diameter less than or equal to $\pi / C$ and suppose $f: \Omega \rightarrow \mathbf{C P}^{n}$ is a locally biholomorphic mapping. For $p \in \Omega$, let $P=f(p)$. Suppose the operators $\mathcal{S}$ and $L$ satisfy the following inequalities for all $Y \in T^{1,0} \Omega$ with $\|Y\|=1$.

1. $|\operatorname{Re} L(Y, Y)| \leq C$,

2. $\operatorname{Re}(\mathcal{S}(Y, Y))\left(U_{P}\right) \geq 0$ for all $P \in f(\Omega)$, where $U_{P}^{2}=\left(W_{P} \circ f\right)$. $\left\|f^{*} v_{F S}\right\|^{1 /(n+1)}$.

Then $f$ is a univalent on $\Omega$.

Proof. First choose a set of nonhomogeneous coordinates $\left[Z^{0}: \ldots: Z^{n}\right]$ on $\mathbf{C P}^{n}$ such that the point $P$ corresponds to $[1: 0: \ldots: 0]$. Then the function $W_{P}$ has the representation

$$
W_{P}\left(Z^{0}, \ldots, Z^{n}\right)=\frac{\left|Z^{1}\right|^{2}+\ldots+\left|Z^{n}\right|^{2}}{\left|Z^{0}\right|^{2}+\ldots+\left|Z^{n}\right|^{2}} .
$$

Let $\varsigma_{i}=Z^{i} / Z^{0}$ be nonhomogeneous coordinates in a neighborhood of $P$. With respect to these coordinates, $f: \Omega \rightarrow \mathbf{C P}^{n}$ has the representation $f(z)=\left(f^{1}(z), \ldots, f^{n}(z)\right)$ with $f(p)=(0, \ldots, 0)$. Since $\Sigma$ and $L$ are invariant under Euclidean changes of coordinates on the domain $\Omega$, we may assume $p=(0, \ldots, 0)$ in a Euclidean coordinate system on $\Omega$. Let

$$
V=W_{P} \circ f \cdot\left\|f^{*} v_{F S}\right\|^{1 /(n+1)} .
$$

The canonical lift of $f$ with respect to the representation $f=\left(f^{1}, \ldots, f^{n}\right)$ is given by $\tilde{f}=\left(\tilde{f}^{0}, \ldots, \tilde{f}^{n}\right)=\left(\lambda, \lambda f^{1}, \ldots, \lambda f^{n}\right)$. In terms of the canonical lift, $V$ is given by

$$
V=c\left(\left|\lambda f^{1}\right|^{2}+\ldots+\left|\lambda f^{n}\right|^{2}\right)
$$

where $c$ is a constant that depends only on $n$.

Now Equation (2.1) may be rewritten as

$$
\frac{\partial^{2}\left(\tilde{f}^{\alpha}\right)}{\partial z^{j} \partial z^{k}}=-L_{j k} \cdot\left(\tilde{f}^{\alpha}\right)+\mathcal{S}_{j k}^{i} \frac{\partial \tilde{f}^{\alpha}}{\partial z^{i}}
$$

where $\tilde{f}^{\alpha}=\lambda f^{\alpha}$.

We also need the following expression for the mixed derivatives of $V$.

$$
\frac{\partial^{2} V}{\partial z^{i} \partial \bar{z}^{j}}=\frac{1}{V} \frac{\partial V}{\partial z^{i}} \frac{\partial V}{\partial \bar{z}^{j}}+V \frac{\partial^{2} \log V}{\partial z^{i} \partial \bar{z}^{j}} .
$$

Now let $\gamma(t)$ be a geodesic parameterized by arclength through $p$ with $\gamma(0)=$ $p=0$. Let $X=\dot{\gamma}(t)$ denote the tangent vector field of $\gamma$. Let $Y$ denote 
the $(1,0)$ component of $X$ so $X=Y+\bar{Y}$. In the $z$ coordinates on $\Omega$, $Y=Y^{\ell} \partial / \partial z^{\ell}$. We compute the second derivative of $V$ along $\gamma(t)$ via the Hessian of $V$, defined as $\operatorname{Hess}(V)=\nabla d(V)$.

$$
\ddot{V}(t)=\operatorname{Hess} V(X, X)=\operatorname{Hess} V(Y, Y)+2 \operatorname{Hess} V(Y, \bar{Y})+\operatorname{Hess} V(\bar{Y}, \bar{Y}) \text {. }
$$

Now using the computations for the partial derivatives for $V$ given by (3.1) and (3.2) we obtain the differential equation

$$
\ddot{V}(t)+2 \operatorname{Re} L(Y, Y) \cdot V(t)=2 \operatorname{Re} \mathcal{S}^{k}(Y, Y) \frac{\partial V}{\partial z^{k}}+2 Y^{\ell} \bar{Y}^{j} \frac{\partial^{2} V}{\partial z^{\ell} \partial \bar{z}^{j}} .
$$

Note that for a vector field $Y$ of type $(1,0), \mathcal{S}(Y, Y)$ is a vector field of type $(1,0)$ and may be written with respect to the $z^{k}$ coordinate system as $\mathcal{S}(Y, Y)=\mathcal{S}^{k}(Y, Y) \partial / \partial z^{k}$. Since $\dot{V}(t)=X(V)=2 \operatorname{Re} \partial V(Y)$ we have the inequality

$$
\partial V(Y) \bar{\partial} V(\bar{Y}) \geq \frac{1}{4}(\dot{V})^{2} .
$$

Using Equation (3.2) in Equation (3.3) this gives the inequality

$$
\begin{aligned}
& \ddot{V}+2 \operatorname{Re} L(Y, Y) \cdot V \geq \\
& 2[\operatorname{Re} \mathcal{S}(Y, Y)](V)+\frac{1}{2} \frac{(\dot{V})^{2}}{V}+V \partial \bar{\partial} \log V(Y, \bar{Y}) .
\end{aligned}
$$

Note that on the right hand side of the above inequality, the first term is a vector field applied to a function. We also note that the inequality fails to be sharp by the term $|\operatorname{Im} \partial V(Y)|^{2}$ on the right hand side.

If we make the dependent change of variable $U^{2}=V$ we obtain the differential inequality

$$
\ddot{U}+\operatorname{Re} L(Y, Y) \cdot U \geq 2[\operatorname{Re} \mathcal{S}(Y, Y)](U)+2 U \cdot \partial \bar{\partial} \log U(Y, \bar{Y}) .
$$

Since $U^{2}=\left|\lambda f^{1}\right|^{2}+\ldots+\left|\lambda f^{n}\right|^{2}$ the last term on the right hand side is non-negative. By the assumption on $\mathcal{S}$ we have

$$
\ddot{U}+\operatorname{Re} L(Y, Y) \cdot U \geq 0 \text {. }
$$

Since $U(0)=0$ and $U(\gamma(t))>0$ for small $t$ (because $f$ is locally biholomorphic by hypothesis), the assumption $|\operatorname{Re} L(Y, Y)| \leq C$ and the Sturm comparison theorem imply that $U$ cannot vanish in the interval $0<t<\pi / C$. Hence $f$ cannot be zero in the ball of radius $\pi / C$ except at $p$. 
Corollary. Suppose $f: \Omega \rightarrow \mathbf{P}^{n} \mathbf{C}$ has constant Jacobian in $\Omega$ and $\operatorname{Re}(\mathcal{S}(Y, Y))(U) \geq 0$ for all $Y \in T^{1,0} \Omega$. Then $f$ is univalent in $\Omega$.

Proof. If $f$ has constant Jacobian in $\Omega$, then an easy computation [4] shows that $L(Y, Y) \equiv 0$. Hence condition (1) of the theorem is satisfied for all $C>0$ and the theorem says that $f$ is univalent on $\Omega \cap B(R)$ where $R$ is arbitrarily large.

\section{Example.}

Finally we consider an example, compute the operators $\mathcal{S}$ and $L$, and write down the differential equation for the function $V$ from Equation (3.3). Although we have collected results in Theorem 3.1, the role of the operators $\mathcal{S}$ and $L$ in the question of univalence is perhaps best understood via Equation (3.3). We will show that for this example, Equation 3.3 yields better results than does simply applying Theorem 3.1, but even these are not optimal univalence results.

Let

$$
f(z, w)=\left(f^{1}(z, w), f^{2}(z, w)\right)=\left(w e^{z}, e^{-z}-1\right) .
$$

In the computation of $\mathcal{S}$ from (2.1), we identify $z^{1}=z$ and $z^{2}=w$. We want to write down Equation (3.3) for real lines through (0,0). Since the Jacobian determinant is constant, the canonical lift, $\tilde{f}$, is given by

$$
\tilde{f}(z, w)=\left(1, f^{1}(z, w), f^{2}(z, w)\right) .
$$

Since $\lambda=1$, a straightforward computation using (2.2) shows that $L=0$. Also, (2.1) gives the expression for $\mathcal{S}$,

$$
\mathcal{S}_{i j}^{k}=\left[\left(\frac{\partial f}{\partial z}\right)^{-1}\right]_{l}^{k} \frac{\partial^{2} f^{l}}{\partial z^{i} z^{j}} .
$$

The function $V$ is given by (up to a constant multiple)

$$
V(z, w)=\left(\left|w e^{z}\right|^{2}+\left|e^{-z}-1\right|^{2}\right)
$$

Using (4.1) and (4.2) we see that

$$
\mathcal{S}(V)=\left(\left|w e^{z}\right|^{2}+\left|e^{-z}\right|^{2}-e^{-z}\right) d z \otimes d z+\bar{w}\left|e^{z}\right|^{2}(d z \otimes d w+d w \otimes d z) .
$$

If we take $Y=\partial / \partial z$ so $Y+\bar{Y}=\partial / \partial x$, we obtain

$$
\mathcal{S}(V)(Y, Y)=\left|w e^{z}\right|^{2}+\left|e^{-z}\right|^{2}-e^{-z} .
$$


We now verify Equation (3.3).

Compute $Y \bar{Y}(V)=\frac{\partial^{2} V}{\partial z \partial \bar{z}}$ and evaluate along the complex line $\{(z, 0): z \in \mathbf{C}\}$.

$$
\left.\frac{\partial^{2} V}{\partial z \partial \bar{z}}\right|_{w=0}=\left|e^{-z}\right|^{2}
$$

Hence

$$
2 \operatorname{Re} \mathcal{S}(V)(Y, Y)+2 Y \bar{Y}(V)=2\left(2\left|e^{-z}\right|^{2}-\operatorname{Re}\left(e^{-z}\right)\right) .
$$

If we evaluate this expression along the real line $l=\{((x, 0), 0): x \in \mathbf{R}\} \subset$ $\mathbf{C}^{2}$ we obtain,

$$
\left.\{2 \operatorname{Re} \mathcal{S}(V)(Y, Y)+2 Y \bar{Y}(V)\}\right|_{l}=2 e^{-x}\left(2 e^{-x}-1\right) .
$$

This is just the right side of Equation (3.3). On the other hand if we restrict $V$ to the line $l$ we obtain $\left.V\right|_{l}=\left(e^{-x}-1\right)^{2}$. Thus $V_{x x}=4\left(e^{-x}\right)^{2}-2 e^{-x}$, and we have verified Equation (3.3) for this example. One easily sees that $\operatorname{Re}(\mathcal{S}(Y, Y))(U) \geq 0$ for $x \leq 0$, so, using the method of the proof of Theorem 3.1, we obtain univalence along the ray $\{((x, 0), 0): x \leq 0, x \in \mathbf{R}\}$ originating at $(0,0)$. On the other hand if we use the positive contribution of the second term in the right side of (3.3), we obtain univalence on $\{((x, 0), 0): x<\log 2\}$. Clearly this is not optimal; one can see directly that $f$ is in fact univalent on the whole line $l$. We note that the statement of Theorem 3.1 yields nothing for this example: Although condition 1 is trivially true, condition 2 does not hold in any neighborhood of 0 .

We remark again that our object in this paper has been to isolate natural differential operators associated with a holomorphic mapping that are useful in obtaining univalence criteria. Equation (3.3) shows how the operators $\mathcal{S}$ and $L$ serve this purpose.

\section{References}

[1] F.G. Avkhadiev and L.A. Aksent'ev, The main results on sufficient conditions for an analytic function to be schlicht, Russian Math. Surveys, 30 (1975), 1-64.

[2] Keith Carne, The Schwarzian derivative for conformal maps, Journal reine u. angewandte Math., 408 (1990), 10-33.

[3] Martin Chuaqui, A unified approach to univalence criteria in the unit disc and simply connected domains, preprint.

[4] Robert Molzon and Karen Pinney Mortensen, Differential operators associated with holomorphic mappings, Annals of Global Analysis and Geometry, 12 (1994), 291304.

[5] _ A characterization of complex projective space up to biholomorphic isometry, to appear in Journal of Geometric Analysis. 
[6] Z. Nehari, Univalence criteria depending on the Schwarzian derivative, Ill. J. Math., 23 (1979), 345-351.

[7] B. Osgood and D. Stowe, The Schwarzian derivative and conformal mappings of Riemannian manifolds, Duke Math. J., 67 (1992), 57-99.

[8] J.A. Pfaltzgraff, Subordination chains and univalence of holomorphic mappings in $C^{n}$, Math. Ann., 210 (1974), 55-68.

[9] Shin-Iohi Takahashi, Univalent mappings in several complex variables, Annals of Math., 53 (1951), 464-471.

Received August 30, 1994 and revised December 20, 1994. The second author was partially supported by the National Science Foundation.

UNIVERSITY OF KENTUCKY

LeXington, KentuCKY 40506

E-mail address: pinney@ms.uky.edu 